\title{
Students Evaluate Online Teaching
}

\section{A survey of physics students pinpoints strategies for improving the online teaching experience.}

\author{
By Michael Schirber
}

S tudents around the world have been forced to adapt to new teaching methods developed in response to the COVID-19 pandemic. A new study has surveyed university students in physics classes to see how they are coping with distance learning [1]. The results show that self-organization is an essential ingredient for adapting to the new teaching environment. For students that are struggling, the researchers have several suggestions, such as hosting live problem-solving sessions and prioritizing first-year students for on-campus teaching. To better motivate students, they also recommend emphasizing some of the positive aspects of distance learning, such as the opportunity to be more creative with laboratory assignments.

In the spring of 2020, many universities around the world closed

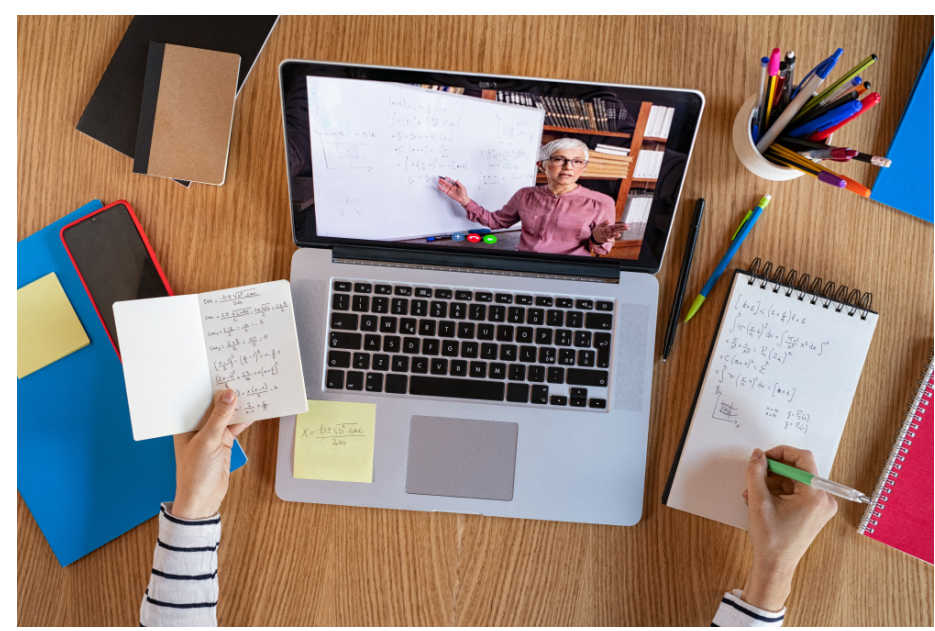

Distance learning has required adjustments from teachers and students.

Credit: Rido/stock.adobe.com their doors and switched to online courses. A group of teachers in Europe immediately began coordinating with each other to find the best teaching methods. "All of us were giving lectures or courses, and we had to prepare for the new situation," says Pascal Klein from the University of Göttingen in Germany. He and his colleagues shared ideas, such as how to organize at-home experiments and which video-conference software to use. But it was hard to tell what was working. "We often felt like we were talking to a wall, since very few students switched on their cameras," says Ana Susac from the University of Zagreb in Croatia.

Susac, Klein, and their colleagues decided to perform a study to gauge the effectiveness of online teaching. They devised an online questionnaire that asked students to assess their own learning behavior and rate learning achievements during lockdowns. The advantage of self-reporting over objective evaluations or exams is that it poses less of a time burden on the students, Klein says. "Also, students feel less like they are being tested and more like their opinions are valued." Nearly 600 physics students at five European universities completed the questionnaire. Because the survey is based on subjective perceptions, the researchers could not make quantitative assessments of teaching effectiveness, but they were able to draw insights by correlating the responses of individual students.

They found that students who considered themselves well-organized were often the ones who felt most at ease with distance learning. "Self-organization is always an important factor in learning," Klein says. "But in the online environment, it seems even more important to be able to organize your day, to set routines, and to motivate yourself." The students that gave 
themselves low marks on self-organization were the ones that felt least comfortable with online teaching. The main reason seems to be the decrease in interactions with professors and other students. "While the excellent students might not care too much, this reduced communication certainly has an impact on the medium-to-poor achieving students, who will fall further behind," Klein says.

Bethany Wilcox from the University of Colorado, Boulder-who was not involved with the study-believes the results are consistent with the situation in many American universities during COVID-19. "Remote learning is very challenging for students and demands a level of motivation and self-direction that many adults do not have," she says. She and her colleagues performed a similar study of students' experiences in the US and found the same trends as those found by Klein and co-workers in Europe. However, she noted that compared with European students, a larger fraction of American students cited difficulties in finding a reliable internet connection and a quiet study space.

Klein and his colleagues have several recommendations for improving student performance during the pandemic. For example, they stress the need for doing "live" online problem-solving sessions, so that students can give and get feedback. For universities that open partially (following the so-called "hybrid" approach), the researchers recommend giving priority to first-year students for in-class teaching, as the data suggested that they suffer the most from reduced interaction. And because students' attitudes toward teaching correlate with achievement, the team suggests emphasizing the positive aspects of distance learning. One benefit, Susac says, is that performing experiments at home-as opposed to a controlled lab setting-has given students the opportunity to be more creative in putting together equipment and collecting data. "We have been pleasantly surprised with the nice setups and good questions that the students have come up with," she says.

Many of the students in the survey said that they were pleased with the level of online teaching, but coauthor Lana Ivanjek from TU Dresden in Germany wonders if that was mostly a result of low expectations. "At the beginning, I think that students were very satisfied with how things were working because they were happy that it worked at all," Ivanjek says. But now everyone is more accustomed to distance learning. "We teachers are better prepared, but the students also have higher expectations for the quality of teaching," she says.

Michael Schirber is a Corresponding Editor for Physics based in Lyon, France.

\section{REFERENCES}

1. P. Klein, "Studying physics during the COVID-19 pandemic: Student assessments of learning achievement, perceived effectiveness of online recitations, and online laboratories," Phys. Rev. Phys. Educ. Res. 17, 010117 (2021). 